PROCEEDINGS OF THE

AMERICAN MATHEMATICAL SOCIETY

Volume 136, Number 12, December 2008, Pages 4305-4312

S 0002-9939(08)09396-9

Article electronically published on June 30,2008

\title{
DELAY-DEPENDENT AND DELAY-INDEPENDENT STABILITY CRITERIA FOR A DELAY DIFFERENTIAL SYSTEM
}

\author{
HIDEAKI MATSUNAGA \\ (Communicated by Carmen C. Chicone)
}

\begin{abstract}
For a linear delay differential system with two coefficients and one delay, we establish some necessary and sufficient conditions on the asymptotic stability of the zero solution, which are composed of delay-dependent and delay-independent stability criteria. On the former criterion, the range of the delay is explicitly given.
\end{abstract}

\section{INTRODUCTION}

In this paper we study the asymptotic stability of a linear delay differential system with two coefficients and one delay

$$
x^{\prime}(t)=-a x(t)-B x(t-\tau), \quad t \geq 0,
$$

where $a$ is a real number, $B$ is a $2 \times 2$ real constant matrix, and $\tau$ is a positive number. The stability problem of (1) is determined completely by the roots of its associated characteristic equation

$$
F(\lambda) \equiv \operatorname{det}\left(\lambda I+a I+B e^{-\lambda \tau}\right)=0,
$$

where $I$ is the $2 \times 2$ identity matrix. It is well-known that the zero solution of (1) is asymptotically stable if and only if all the roots of (2) lie in the left half of the complex plane (see [4]).

In the scalar case, system (1) is expressed as

$$
x^{\prime}(t)=-a x(t)-b x(t-\tau), \quad t \geq 0,
$$

where $b$ is a real number. In [6], Hayes gave the stability criterion for (3) as follows:

Theorem A. The zero solution of (3) is asymptotically stable if and only if

$$
a>-\frac{1}{\tau}, \quad a+b>0 \quad \text { and } \quad b<\omega \sin \omega \tau-a \cos \omega \tau,
$$

where $\omega$ is the solution in $(0, \pi / \tau)$ of $\omega \cos \omega \tau=-a \sin \omega \tau$.

Received by the editors October 19, 2007.

2000 Mathematics Subject Classification. Primary 34K20; Secondary 34K25.

Key words and phrases. Asymptotic stability, stability criteria, delay differential equations, characteristic equation.

This work was supported in part by Grant-in-Aid for Young Scientists (B), No. 19740071, of the Japanese Ministry of Education, Culture, Sports, Science and Technology.

(C)2008 American Mathematical Society Reverts to public domain 28 years from publication 


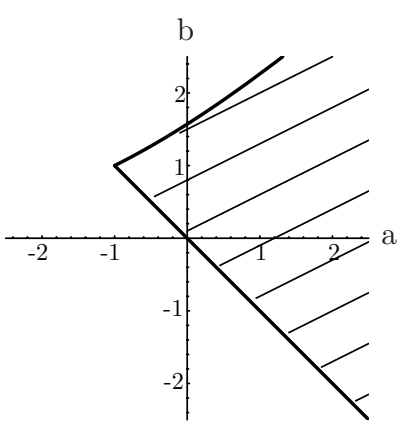

FIgURE 1. Stability region of (3) with $\tau=1$

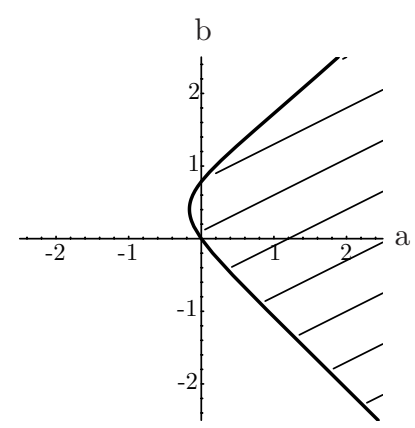

FIGURE 2. Stability region of (11) with $\tau=1$ and $\theta=\pi / 4$

The stability region which means the set of all $(a, b)$ in which the zero solution of (31) is asymptotically stable is presented by the region in Figure 1 (cf. 44, p. 135]). The upper boundary of the stability region of (3) is given parametrically by the equation

$$
a=-\frac{\omega}{\tan \omega \tau}, \quad b=\frac{\omega}{\sin \omega \tau}, \quad 0<\omega<\frac{\pi}{\tau} .
$$

Recently, in 10, Sakata has extended Theorem A to system (11).

Theorem B. Let be $e^{ \pm i \theta}$ be eigenvalues of $B$ where $b$ and $\theta$ are real numbers with $|\theta| \leq \pi / 2$. Then the zero solution of (11) is asymptotically stable if and only if

$$
\begin{gathered}
a>\phi(b), \quad \text { if } \theta \neq 0, \\
a+b>0 \quad \text { and } a>\phi(b), \quad \text { if } \theta=0,
\end{gathered}
$$

where the curve $a=\phi(b)$ is given parametrically by the equation

$$
a=-\frac{\omega}{\tan (\omega \tau-|\theta|)}, \quad b=\frac{\omega}{\sin (\omega \tau-|\theta|)}, \quad-\frac{\pi-|\theta|}{\tau}<\omega<\frac{|\theta|}{\tau} .
$$

The stability region of (1) with fixed $\tau$ and $\theta$ is presented by the region in Figure 2. A natural question now arises: how does the asymptotic stability of (11) with fixed $a$ and $B$ depend on the delay $\tau$ ? The purpose of this paper is to answer the question. It should be pointed out that the range of $\tau$ with fixed $a$ and $B$ for the zero solution of (11) to be asymptotically stable cannot be derived explicitly from Theorem B.

Several authors ([1, 2], 3]) examined the above question for the scalar case. By virtue of their work, we have another type of the stability criterion for (3).

Theorem C. The zero solution of (3) is asymptotically stable if and only if either

$$
a+b>0 \quad \text { and } \quad b^{2}-a^{2} \leq 0
$$

$$
a+b>0, \quad b^{2}-a^{2}>0 \quad \text { and } \quad \tau<\frac{1}{\sqrt{b^{2}-a^{2}}} \arccos \left(-\frac{a}{b}\right) .
$$

We notice that condition (4) is equivalent to $-a<b \leq a$ and is independent of $\tau$; and so, it is the stability criterion for all $\tau>0$. On the other hand, condition (5) depends on $\tau$; more precisely, the range of $\tau$ is explicitly given. Our goal is to extend Theorem $\mathrm{C}$ to system (1). 


\section{MAin Results}

By the transfomation $x(t)=P u(t)$ with a nonsingular matrix $P$, system (11) can be written as

$$
u^{\prime}(t)=-a u(t)-P^{-1} B P u(t-\tau), \quad t \geq 0,
$$

and therefore, we have only to consider (1), where the matrix $B$ is either of the following two matrices in Jordan form:

$$
\text { (I) } B=\left(\begin{array}{cc}
b_{1} & c \\
0 & b_{2}
\end{array}\right), \quad \text { (II) } B=b R(\theta) \equiv b\left(\begin{array}{cc}
\cos \theta & -\sin \theta \\
\sin \theta & \cos \theta
\end{array}\right),
$$

where $b_{1}, b_{2}, c, b$ and $\theta$ are real numbers with $|\theta| \leq \pi / 2$.

We first consider system (1), where $B$ is given by (I); that is,

$$
x^{\prime}(t)=-a x(t)-\left(\begin{array}{cc}
b_{1} & c \\
0 & b_{2}
\end{array}\right) x(t-\tau) .
$$

By (2), the characteristic equation of (6) becomes

$$
\left(\lambda+a+b_{1} e^{-\lambda \tau}\right)\left(\lambda+a+b_{2} e^{-\lambda \tau}\right)=0 .
$$

Since $\lambda+a+b_{k} e^{-\lambda \tau}=0$ is the characteristic equation of (3) with $b=b_{k}$ for $k=1,2$, one can immediately obtain the following result from Theorem C.

Theorem 1. The zero solution of (6) is asymptotically stable if and only if for $k=1,2$, either

$$
a+b_{k}>0 \quad \text { and } \quad b_{k}^{2}-a^{2} \leq 0
$$

or

$$
a+b_{k}>0, \quad b_{k}^{2}-a^{2}>0 \quad \text { and } \quad \tau<\frac{1}{\sqrt{b_{k}^{2}-a^{2}}} \arccos \left(-\frac{a}{b_{k}}\right) .
$$

Remark 1. Theorem 1 shows that the stability criterion for (6) is independent of $c$.

We next consider system (1), where $B$ is given by (II); that is,

$$
x^{\prime}(t)=-a x(t)-b R(\theta) x(t-\tau) .
$$

The following theorem is our main result.

Theorem 2. The zero solution of (17) is asymptotically stable if and only if either

$$
a+b \cos \theta>0 \quad \text { and } \quad b^{2}-a^{2} \leq 0
$$

or

(9) $a+b \cos \theta>0, \quad b^{2}-a^{2}>0 \quad$ and $\tau<\frac{\operatorname{sgn}(b)}{\sqrt{b^{2}-a^{2}}}\left\{\arccos \left(-\frac{a}{b}\right)-|\theta|\right\}$.

Remark 2. Condition (8) is equivalent to $-a<b \leq a$ if $\theta=0$; $|b| \leq a$ if $0<|\theta| \leq$ $\pi / 2$.

Remark 3. In case $a=0$, system (7) becomes

$$
x^{\prime}(t)=-b R(\theta) x(t-\tau) .
$$

Theorem 2 then asserts that the stability criterion for (10) is

$$
0<b \tau<\frac{\pi}{2}-|\theta|
$$

which coincides with Theorem 3.3 in [5] or Lemma 1 in [9]. 
Remark 4 . In case $\theta=0$, system (17) is reduced to the scalar equation

$$
x^{\prime}(t)=-a x(t)-b x(t-\tau) .
$$

In this case, Theorem 2 coincides with Theorem C.

Remark 5 . In case $\theta=\pi / 2$, system (7) becomes the system with off-diagonal delays

$$
\left\{\begin{array}{l}
x^{\prime}(t)=-a x(t)+b y(t-\tau), \\
y^{\prime}(t)=-b x(t-\tau)-a y(t) .
\end{array}\right.
$$

Theorem 2 together with Remark 2 then asserts that the stability criterion for (11) is either

$$
a \geq|b|
$$

or

$$
a>0 \quad \text { and } \quad \tau<\frac{\operatorname{sgn}(b)}{\sqrt{b^{2}-a^{2}}}\left\{\arccos \left(-\frac{a}{b}\right)-\frac{\pi}{2}\right\}=\frac{\arccos \left(1-2 a^{2} / b^{2}\right)}{2 \sqrt{b^{2}-a^{2}}} .
$$

We notice that the latter condition depending on $\tau$ cannot be given by Theorem 1 in [7.

In order to prove Theorem 2, we will investigate the distribution of the roots of the characteristic equation of (7). For simplicity, let $f(\lambda) \equiv \lambda+a+b e^{-\lambda \tau+i|\theta|}$. Then, by (2), we have

$$
\begin{aligned}
F(\lambda) & =\left|\begin{array}{cc}
\lambda+a+b e^{-\lambda \tau} \cos \theta & -b e^{-\lambda \tau} \sin \theta \\
b e^{-\lambda \tau} \sin \theta & \lambda+a+b e^{-\lambda \tau} \cos \theta
\end{array}\right| \\
& =\left(\lambda+a+b e^{-\lambda \tau} \cos \theta\right)^{2}+\left(b e^{-\lambda \tau} \sin \theta\right)^{2} \\
& =\left(\lambda+a+b e^{-\lambda \tau} \cos |\theta|\right)^{2}-\left(i b e^{-\lambda \tau} \sin |\theta|\right)^{2} \\
& =\left(\lambda+a+b e^{-\lambda \tau+i|\theta|}\right)\left(\lambda+a+b e^{-\lambda \tau-i|\theta|}\right) \\
& =f(\lambda) \overline{f(\bar{\lambda})},
\end{aligned}
$$

where $\bar{\lambda}$ is the complex conjugate of any complex $\lambda$. Taking note that $\overline{f(\bar{\lambda})}=0$ implies $f(\bar{\lambda})=0$, one can easily see that the following proposition holds.

Proposition 1. The zero solution of (7) is asymptotically stable if and only if all the roots of the equation

$$
f(\lambda) \equiv \lambda+a+b e^{-\lambda \tau+i|\theta|}=0
$$

lie in the left half of the complex plane.

Since $f(\lambda)$ is an analytic function of $\lambda$ and $\tau$ for fixed $a, b$ and $\theta$, one can regard the root $\lambda=\lambda(\tau)$ of (12) as a continuous function of $\tau$. The next proposition established by Theorem 2.4 in [3] plays an essential role in the proof of Theorem 2 (see also [8, Chapter 3]).

Proposition 2. As $\tau$ varies, the sum of the multiplicities of the roots of (12) in the open right half-plane can change only if a root appears on or crosses the imaginary axis.

Consequently, we will determine the value of $\tau$ at which equation (12) may have roots on the imaginary axis. 
Lemma 1. Assume that $0<\theta \leq \pi / 2$ and $b>0$. Let $i \omega$ be a root of (12) where $\omega$ is a real number. Then the following statements hold:

(i) In case $b^{2}-a^{2} \leq 0$, there exists no real number $\omega$; that is, equation (12) has no root on the imaginary axis for all $\tau>0$.

(ii) In case $b^{2}-a^{2}>0$, the real number $\omega$ and the delay $\tau$ are expressed as

$$
\omega= \pm \sqrt{b^{2}-a^{2}} \text { and } \tau=\tau_{n}^{ \pm},
$$

for $n=0,1,2, \ldots$, where $\tau_{n}^{+}$and $\tau_{n}^{-}$are given by

$$
\begin{aligned}
& \tau_{n}^{+}=\frac{1}{\sqrt{b^{2}-a^{2}}}\left\{2 n \pi+\arccos \left(-\frac{a}{b}\right)+\theta\right\}, \\
& \tau_{n}^{-}=\frac{1}{\sqrt{b^{2}-a^{2}}}\left\{2 n \pi+\arccos \left(-\frac{a}{b}\right)-\theta\right\} .
\end{aligned}
$$

Conversely, if $\tau=\tau_{n}^{+}$or $\tau=\tau_{n}^{-}$, then $i \sqrt{b^{2}-a^{2}}$ or $-i \sqrt{b^{2}-a^{2}}$ is a root of (12).

Proof. We first notice that $\omega \neq 0$ because $f(0)=a+b \cos \theta+i b \sin \theta \neq 0$. If $f(i \omega)=0$, then $i \omega+a+b e^{-i(\omega \tau-\theta)}=0$ or, equivalently,

$$
b \cos (\omega \tau-\theta)=-a \text { and } b \sin (\omega \tau-\theta)=\omega .
$$

By squaring both sides of the above equations and adding them together, we have

$$
b^{2}=a^{2}+\omega^{2} .
$$

Therefore, in case $b^{2}-a^{2} \leq 0$, condition (14) implies $\omega^{2} \leq 0$, which contradicts $\omega^{2}>$ 0 ; thus statement (i) is verified. On the other hand, in case $b^{2}-a^{2}>0$, condition (14) implies $\omega= \pm \sqrt{b^{2}-a^{2}}$. If $\omega=\sqrt{b^{2}-a^{2}}$, relation (13) with $\sin (\omega \tau-\theta)>0$ leads to

$$
\omega \tau-\theta=2 n \pi+\arccos (-a / b), \quad n=0,1,2, \ldots,
$$

which yields $\tau=\tau_{n}^{+}$. Also, if $\omega=-\sqrt{b^{2}-a^{2}}$, relation (13) with $\sin (\omega \tau-\theta)<0$ leads to

$$
\omega \tau-\theta=-2 n \pi-\arccos (-a / b), \quad n=0,1,2, \ldots,
$$

which yields $\tau=\tau_{n}^{-}$.

Next, since

$$
\arccos \left(-\frac{a}{b}\right)=\left\{\begin{array}{lll}
\arcsin \left(\sqrt{1-(-a / b)^{2}}\right) & \text { if } \quad a \leq 0, \\
\pi-\arcsin \left(\sqrt{1-(-a / b)^{2}}\right) & \text { if } \quad a>0,
\end{array}\right.
$$

we have $\sin (\arccos (-a / b))=\sqrt{b^{2}-a^{2}} / b$. Therefore, if $\tau=\tau_{n}^{+}$, we obtain

$$
\begin{aligned}
f\left(i \sqrt{b^{2}-a^{2}}\right) & =i \sqrt{b^{2}-a^{2}}+a+b e^{-i\left(\sqrt{b^{2}-a^{2}} \tau_{n}^{+}-\theta\right)} \\
& =i \sqrt{b^{2}-a^{2}}+a+b e^{-i(2 n \pi+\arccos (-a / b))} \\
& =i \sqrt{b^{2}-a^{2}}+a+b\{\cos (\arccos (-a / b))-i \sin (\arccos (-a / b))\} \\
& =i \sqrt{b^{2}-a^{2}}+a+b\left(-a / b-i \sqrt{b^{2}-a^{2}} / b\right)=0,
\end{aligned}
$$

which implies $i \sqrt{b^{2}-a^{2}}$ is a root of (12). Similarly, if $\tau=\tau_{n}^{-}$, one can show that $-i \sqrt{b^{2}-a^{2}}$ is a root of (12). This completes the proof.

When $b<0$, we have the following analogous result.

Lemma 2. Assume that $0<\theta \leq \pi / 2$ and $b<0$. Let $i \omega$ be a root of (12) where $\omega$ is a real number. Then the following statements hold: 
(i) In case $b^{2}-a^{2} \leq 0$, there exists no real number $\omega$; that is, equation (12) has no root on the imaginary axis for all $\tau>0$.

(ii) In case $b^{2}-a^{2}>0$, the real number $\omega$ and the delay $\tau$ are expressed as

$$
\omega= \pm \sqrt{b^{2}-a^{2}} \text { and } \tau=\sigma_{n}^{ \pm},
$$

for $n=0,1,2, \ldots$, where $\sigma_{n}^{+}$and $\sigma_{n}^{-}$are given by

$$
\begin{aligned}
& \sigma_{n}^{+}=\frac{1}{\sqrt{b^{2}-a^{2}}}\left\{2 n \pi-\arccos \left(-\frac{a}{b}\right)+\theta\right\}, \\
& \sigma_{n}^{-}=\frac{1}{\sqrt{b^{2}-a^{2}}}\left\{(2 n+2) \pi-\arccos \left(-\frac{a}{b}\right)-\theta\right\} .
\end{aligned}
$$

Conversely, if $\tau=\sigma_{n}^{+}$or $\tau=\sigma_{n}^{-}$, then $i \sqrt{b^{2}-a^{2}}$ or $-i \sqrt{b^{2}-a^{2}}$ is a root of (12).

Remark 6. In case $b>0$, the definitions of $\tau_{n}^{+}$and $\tau_{n}^{-}$yield

$$
\min \left\{\tau_{n}^{+}, \tau_{n}^{-} \mid n=0,1,2, \ldots\right\}=\left\{\begin{array}{lll}
\tau_{0}^{-} & \text {if } & a+b \cos \theta>0 \\
\tau_{0}^{+} & \text {if } & a+b \cos \theta \leq 0
\end{array}\right.
$$

Similarly, in case $b<0$, the definitions of $\sigma_{n}^{+}$and $\sigma_{n}^{-}$yield

$$
\min \left\{\sigma_{n}^{+}, \sigma_{n}^{-} \mid n=0,1,2, \ldots\right\}=\left\{\begin{array}{lll}
\sigma_{0}^{+} & \text {if } & a+b \cos \theta>0 \\
\sigma_{0}^{-} & \text {if } & a+b \cos \theta \leq 0 .
\end{array}\right.
$$

Furthermore, we will observe how the roots of (12) on the imaginary axis move as $\tau$ increases.

Lemma 3. Assume that $0<\theta \leq \pi / 2$. Then all the roots of (12) on the imaginary axis move in the right half-plane as $\tau$ increases.

Proof. Let $i \omega$ be a root of (12) where $\omega$ is a nonzero real number. It suffices to show $\left.\operatorname{Re}(d \lambda / d \tau)\right|_{\lambda=i \omega}>0$. Taking the derivative of $\lambda$ with respect to $\tau$ on (12), we have

namely,

$$
\frac{d \lambda}{d \tau}-b \lambda e^{-\lambda \tau+i \theta}-b \tau e^{-\lambda \tau+i \theta} \frac{d \lambda}{d \tau}=0
$$

Hence it follows that

$$
\frac{d \lambda}{d \tau}=\frac{b \lambda e^{-\lambda \tau+i \theta}}{1-b \tau e^{-\lambda \tau+i \theta}}=-\frac{\lambda(\lambda+a)}{1+\tau(\lambda+a)} .
$$

$$
\left.\frac{d \lambda}{d \tau}\right|_{\lambda=i \omega}=-\frac{i \omega(i \omega+a)}{1+\tau(i \omega+a)}=-\frac{-\omega^{2}+i \omega a}{1+a \tau+i \omega \tau}=\frac{\left(\omega^{2}-i \omega a\right)(1+a \tau-i \omega \tau)}{(1+a \tau)^{2}+(\omega \tau)^{2}},
$$

which implies

$$
\left.\operatorname{Re} \frac{d \lambda}{d \tau}\right|_{\lambda=i \omega}=\frac{\omega^{2}(1+a \tau)-\omega^{2} a \tau}{(1+a \tau)^{2}+(\omega \tau)^{2}}=\frac{\omega^{2}}{(1+a \tau)^{2}+(\omega \tau)^{2}}>0 .
$$

This completes the proof.

Now we are in a position to prove Theorem 2.

Proof of Theorem 2. By virtue of Proposition 1, we verify that all the roots of (12) lie in the left half-plane if and only if either condition (8) or (9) holds. In case $\theta=0$, Remark 4 implies that Theorem 2 is true, and consequently, we have only to consider (12) with $0<\theta \leq \pi / 2$. 
(Sufficiency) In case $\tau=0$, the root of (12) is only $\lambda=-a-b \cos \theta-i b \sin \theta$ which has a negative real part because of the assumption. By the continuity of the roots with respect to $\tau$, this implies that all the roots of (12) lie in the left half-plane for $\tau>0$ sufficiently small.

We here claim that if either condition (8) or (9) holds, then equation (12) has no root on the imaginary axis. In fact, condition (8) and Lemma 1 assert that our claim is valid for all $\tau>0$. If condition (9) holds, then we have $\tau \neq \tau_{n}^{ \pm}$and $\tau \neq \sigma_{n}^{ \pm}$ for $n=0,1,2, \ldots$, because of Remark 6 and the relation

$$
\frac{\operatorname{sgn}(b)}{\sqrt{b^{2}-a^{2}}}\left\{\arccos \left(-\frac{a}{b}\right)-\theta\right\}=\left\{\begin{array}{lll}
\tau_{0}^{-} & \text {if } & b>0 \\
\sigma_{0}^{+} & \text {if } & b<0
\end{array}\right.
$$

By the contraposition of Lemma 1, our claim is also valid in this case.

From the preceding argument and Proposition 2, we therefore conclude that either condition (8) or (9) implies that all the roots of (12) lie in the left half-plane.

(Necessity) To end the proof, we will show the following contraposition: either

$$
a+b \cos \theta \leq 0
$$

or

$$
b^{2}-a^{2}>0 \quad \text { and } \quad \tau \geq \tau_{0} \equiv \frac{\operatorname{sgn}(b)}{\sqrt{b^{2}-a^{2}}}\left\{\arccos \left(-\frac{a}{b}\right)-\theta\right\}
$$

implies that there exists a root $\lambda^{*}$ of (12) such that $\operatorname{Re} \lambda^{*} \geq 0$.

Suppose that (15) holds. Let $\lambda_{1}(\tau)$ be the branch of the root of (12) satisfying $\lambda_{1}(0)=-a-b \cos \theta-i b \sin \theta$. Then the continuity of $\lambda_{1}(\tau)$ or Lemma 3 yields that $\operatorname{Re} \lambda_{1}(\tau)>0$ for $\tau>0$ sufficiently small. Since $\lambda_{1}(\tau)$ cannot move in the left half-plane crossing on the imaginary axis as $\tau$ increases by Lemma 3, we thus obtain $\operatorname{Re} \lambda_{1}(\tau) \geq 0$ for all $\tau \geq 0$.

Suppose that (16) holds. Let $\lambda_{2}(\tau)$ be the branch of the root of (12) satisfying $\lambda_{2}\left(\tau_{0}\right)=-i \operatorname{sgn}(b) \sqrt{b^{2}-a^{2}}$. Then Lemma 3 yields that $\operatorname{Re} \lambda_{2}(\tau)>0$ for $\tau-\tau_{0}>0$ sufficiently small. Since $\lambda_{2}(\tau)$ cannot move in the left half-plane crossing on the imaginary axis as $\tau$ increases by Lemma 3, we therefore obtain $\operatorname{Re} \lambda_{2}(\tau) \geq 0$ for all $\tau \geq \tau_{0}$.

Finally, we consider a $d$-dimensinal linear delay differential system

$$
x^{\prime}(t)=-a x(t)-B x(t-\tau), \quad t \geq 0
$$

where $a$ is a real number, $B$ is a $d \times d$ real constant matrix, and $\tau$ is a positive number.

Let $b_{k} e^{i \theta_{k}}$ denote eigenvalues of $B$ where $b_{k}$ and $\theta_{k}$ are real numbers with $\left|\theta_{k}\right| \leq$ $\pi / 2$ for $k=1,2, \ldots, d$. Then the characteristic equation of (17) is given by

$$
\prod_{k=1}^{d}\left(\lambda+a+b_{k} e^{-\lambda \tau+i\left|\theta_{k}\right|}\right)=0
$$

and thus one can obtain the following result by applying Theorems 1 and 2 . 
Theorem 3. Let $b_{k} e^{i \theta_{k}}$ be eigenvalues of $B$ with $\left|\theta_{k}\right| \leq \pi / 2$ for $k=1,2, \ldots, d$. Then the zero solution of (17) is asymptotically stable if and only if for $k=$ $1,2, \ldots, d$, either

$$
a+b_{k} \cos \theta_{k}>0 \quad \text { and } \quad b_{k}^{2}-a^{2} \leq 0
$$

or

$$
a+b_{k} \cos \theta_{k}>0, \quad b_{k}^{2}-a^{2}>0 \quad \text { and } \quad \tau<\frac{\operatorname{sgn}\left(b_{k}\right)}{\sqrt{b_{k}^{2}-a^{2}}}\left\{\arccos \left(-\frac{a}{b_{k}}\right)-\left|\theta_{k}\right|\right\} .
$$

\section{REFERENCES}

[1] K. L. Cooke and P. van den Driessche, On zeroes of some transcendental equations, Funkcial. Ekvac., 29 (1986), 77-90. MR865215 (87m:34098)

[2] K. L. Cooke and Z. Grossman, Discrete delay, distributed delay and stability switches, J. Math. Anal. Appl., 86 (1982), 592-627. MR652197 (84b:34107)

[3] H. I. Freedman and Y. Kuang, Stability switches in linear scalar neutral delay equations, Funkcial. Ekvac., 34 (1991), 187-209. MR.1130459 (92m:34183)

[4] J. K. Hale and S. M. Verduyn Lunel, Introduction to Functional Differential Equations, Springer-Verlag, New York, 1993. MR1243878 (94m:34169)

[5] T. Hara and J. Sugie, Stability region for systems of differential-difference equations, Funkcial. Ekvac., 39 (1996), 69-86. MR.1401653 (97c:34154)

[6] N. D. Hayes, Roots of the transcendental equation associated with a certain differencedifferential equation, J. London Math. Soc., 25 (1950), 226-232. MR0036426 (12:106d)

[7] J. Hofbauer and J. W.-H. So, Diagonal dominance and harmless off-diagonal delays, Proc. Amer. Math. Soc., 128 (2000), 2675-2682. MR1707519 (2000m:34171)

[8] Y. Kuang, Delay Differential Equations with Applications in Population Dynamics, Academic Press, Boston, 1993. MR.1218880 (94f:34001)

[9] H. Matsunaga, Exact stability criteria for delay differential and difference equations, Appl. Math. Lett., 20 (2007), 183-188. MR2283908 (2007k:34258)

[10] S. Sakata, Asymptotic stability for a linear system of differential-difference equations, Funkcial. Ekvac., 41 (1998), 435-449. MR:1676882 (2000a:34156)

Department of Mathematical Sciences, Osaka Prefecture University, Sakai 599-8531, JAPAN

E-mail address: hideaki@ms.osakafu-u.ac.jp 